\title{
The Palm IAT: A portable version of the implicit association task
}

\author{
JAMES M. DABBS, JR., JONATHAN F. BASSETT, and NATALIA V. DYOMINA \\ Georgia State University, Atlanta, Georgia
}

\begin{abstract}
The Implicit Association Test(IAT) uses reaction times to measure implicit linkages among concepts or between concepts and attributes. The Palm IAT is a simplified version of the IAT that runs on Palm Pilot or Handspring Visor personal digital assistant devices. The Palm IAT is portable, provides precise measurement, and allows for efficient data collection inside or outside the laboratory. Three studies were conducted to assess its practical usefulness. Study $1(N=12)$ replicated a previous demonstration of more favorable implicit associations for flowers than for insects. Study $2(N=9)$ found individual differences in linking the self-concept to characteristics of agency and communion. Study $3(N=$ 23) found individual differences in linking the self-concept to characteristics of boldness and friendliness. Reliability of the Palm IAT is similar to reliability of the original desktop computer version. Using the Palm IAT, one can study hard-to-reach populations in unusual settings.
\end{abstract}

Implicit cognitionslie outside the realm of ordinary awareness, but they affect conscious thoughts and actions. They comprise unconscious and preconscious affects, biases, impressions, beliefs, and attitudes that presumably arise from a person's experience. Even though the person may be unaware of where these cognitions come from or even of their existence, they are linked in varying degrees of closeness to other cognitions and can influence the way the person thinks. The Implicit Association Test (IAT; Greenwald, McGhee, \& Schwartz, 1998) examines the strength of implicit cognitions by using reaction time (RT) to measure linkages among diverse stimulus categories. Because the IAT employs RTs rather than conscious choices and decisions, it is relatively unaffected by the individual's awareness of being tested.

The basic principle behind the IAT is that any two concepts are linked more or less closely to each other in an individual's mind and the closeness of their linkage is reflected in the degree to which they can evoke similar reactions from that individual. People place different items into the same conceptual category more readily when the items are more closely linked. For example, most people have implicit positive views of flowers and negative views of insects, and because of this, they can more quickly give the same response to good and flower or to bad and insect than they can to bad and flower or to good and insect (Greenwald et al., 1998). The IAT can assess the linkage of various concepts to racial groups, social activities, the self, relationships, and other things.

This work was supported by research funds from Georgia State University. The authors especially thank Larry Jamner for his help and suggestions and Marc Weiss of SingleTap.Com for his support in the design and programming of the Palm IAT. Correspondence should be sent to J. M. Dabbs, Department of Psychology, Georgia State University, Atlanta, GA 30303 (e-mail: jdabbs@gsu.edu).
Although the IAT can be used to study a broad range of topics, its use has been restricted by the need to have a desktop or laptop computer to administer it. Lemm and Banaji (2000) describe a simpler paper-and-pencil version, but this version provides less precise measurement and still requires an experimenter and a relatively controlled testing setting. The paper-and-pencil version also does not allow for discarding outlier RTs to individual items. A more truly portable version could introduce the IAT approach to reallife settings outside the laboratory.

The present paper describes an IAT that runs on a Palm Pilot or Handspring personal digital assistant (PDA) computer. These are relatively inexpensive devices that subjects can carry outside the laboratory for periods of days or weeks. Subjects can take the Palm IAT test on repeated occasions, prompted by signals from the PDA or by the occurrence of key events, as instructed by the researcher.

\section{HARDWARE AND SOFTWARE}

Palm Pilot and Handspring PDA's both use the Palm language and operating system (OS). Basic models cost about $\$ 150$ each, and many potential research subjects are familiar with them. They have been used extensively in field studies of such things as moods, everyday behavior, and reactions to medical treatments (cf. Whalen, Jamner, Henker, \& Delfino, 2001). Other PDAs are available that use other OSs, but the present program will not work on these PDAs.

The Palm IAT is an abbreviated version of the original desktop or Web-based IAT (Greenwald et al., 1998; http://www.yale.edu/implicit), and its format is similar to that of the paper-and-pencil IAT (Lemm \& Banaji, 2000). The program is written in $\mathrm{C}++$, compiled into Palm OS language, and transferred to the PDA from a Macintosh or $\mathrm{PC}$ host computer. The host computer uses a Palm conduit to load new stimulus words into the PDA and download 
subjects' responses to the host computer. The Palm IAT uses fewer stimuli than does the original IAT and does not correct for certain response biases; these limitations may be more or less important, depending on the research question being addressed. The Palm IAT software and a description of its functioning, including a flow chart that provides an overview of the core functionality of the software, are available at the Website www.singletap.com. A general description is provided in the next paragraph.

The program displays stimulus words one at a time in the center of the PDA screen, and the subject responds by pressing the left or the right side of the screen as quickly as possible to indicate whether the stimulus word represents a concept displayed on the left or the right side. The screen is set to maximum brightness to maximize readability. Users start the program by tapping the Palm IAT icon on the PDA home screen with a stylus, which causes the program to take control of the PDA, suspend other PDA operations, and display Screen 1 (see Figure 1). As will be described below, subjects operate the program by tapping the screen with a stylus, although the system also works if subjects use their thumbs or fingers to press the screen, and Kip Williams (personal communication) reports similar results when subjects use stylus taps or thumb presses. Subjects enter their initials or other identifying information on Screen 1, using the stylus and an on-screen key pad, and then tap "Continue." Screen 2 appears, with two concept words (shown in uppercase) at the upper left side and two at the upper right side and with three stimulus exemplar words (shown in lowercase) below each concept word. After examining this screen, the subject taps “Continue," and Screen 3 appears, displaying the four concept words alone. The subject taps "Begin Test," and the display changes to Screen 4, with stimulus words beginning to reappear one at a time at the bottom of the screen. The subject presses the left or the right side as quickly as possible after each stimulus word appears, to indicate whether the word represents a concept shown on the left or the right side. The subject can make these responses either by using the stylus or by holding the PDA with the fingers of both hands and using the left and right thumbs to press the left or right side. As soon as the subject responds, the stimulus word disappears, the program records the subject's RT, and a new stimulus word appears $400 \mathrm{msec}$ later. The subject continues through 24 such RT trials, in which stimulus words are presented in a quasi-random order. The order is one selected randomly from a set of 20 predetermined orders, each of which has stimulus words randomized, with the following constraints: Words are selected alternately from among the top six and the bottom six (see Screen 2); words representing each set of three on the left or right side are not used more than four times in a row, and each word is used exactly twice. At the end of 24 trials, Screen 2 reappears, displaying a new set of words from the input file; or if there are no more words in the input file, the program stops and waits for a user to begin a new testing session. At the beginning or end of an IAT session, the experimenter can access Screen 5 by ex- ecuting a power swipe (dragging a stylus from the bottom to the top of the screen). From Screen 5, the experimenter can end the program and return to the PDA home screen, return to Screen 1 to continue with the IAT, or enter a password and go to Screen 6 to designate up to three times per day that the PDA will turn itself on, beep to signal the subject, activate Screen 1, and wait for the subject to begin responding.

IAT stimulus words are uploaded to the PDA from a host Macintosh or PC computer using Palm desktop and conduit software. Table 1 shows a sample input file. The input file consists of lines that each contain four concept words and three stimulus exemplar words for each concept. The words in each line are separated by commas and ordered as they will appear in the upper left, upper right, lower left, and lower right quadrants of the PDA screen. Each IAT requires two input lines, with the words sorted to produce more compatible pairings in one line and less compatible pairings in the other. For example, an IAT on favorability of attitudes toward flowers and insects might have an input file with words arranged to pair flowergood and insect-bad in one line and flower-bad and insectgood in the other, and an IAT on racial prejudice might have words in an input file arranged to pair white-bad and black-good in one line and white-good and black-bad in the other. The input file can include as many lines as desired, with two lines for each IAT to be administered in each testing session.

Subjects' responses are stored in the PDA and sent to the host computer, using the conduit software mentioned above. Table 2 shows a sample data output file. Each line in the output file contains, in tab-delimited format, the subject's initials, the four concept words in the order in which they appeared in the input file, the date and time the test was taken, a number indicating which of 20 preselected random orders was used to present the stimuli, 24 RT scores recorded in 10-msec units, and 24 scores (recorded as 0 or 1 ) indicating whether a response was correct or incorrect. The time-stamping feature is useful in ascertaining the extent to which subjects follow instructions to take the test on specific days and times, information that is needed if one wishes to study data gathered across times representative of a subject's daily life (Schiffman, 2000).

\section{SAMPLE STUDIES}

The three studies below illustrate the usefulness and reliability of the Palm IAT. Study 1 replicates a previous demonstration of implicit preferences for flowers over insects (Citrin \& Greenwald, 1998; Greenwald et al., 1998). Study 2 examines linkages of the self to agency and communion, and Study 3 examines linkages of the self to boldness and hostility.

The subjects in all the studies were college students participating in return for pay or course credit. They met with experimenters who explained the procedure, asked them to read and sign consent forms, administered a practice 


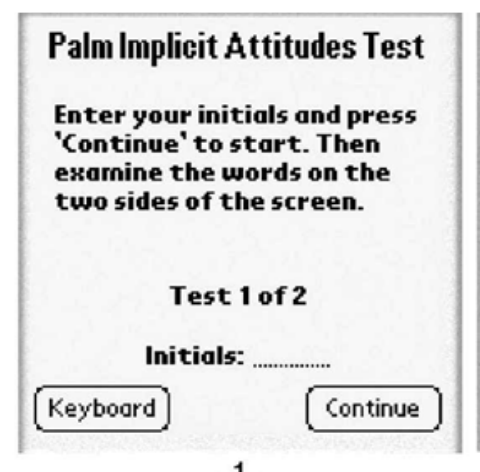

$-1-$

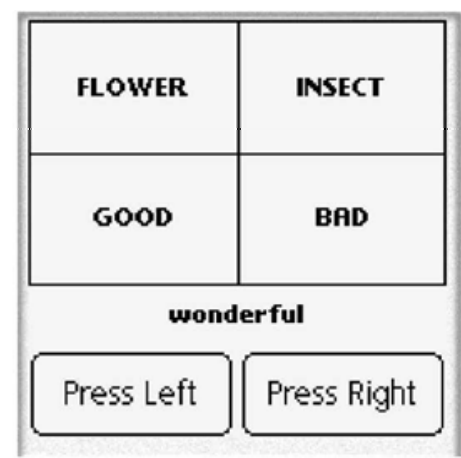

$-4-$

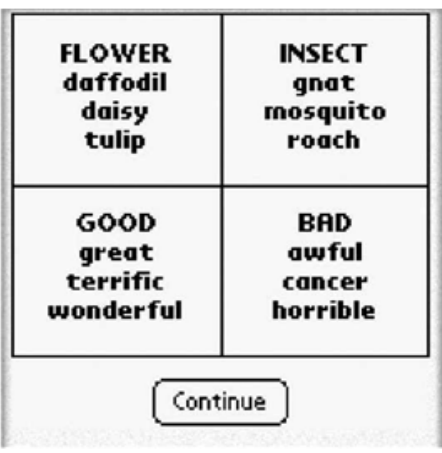

$-2-$



$-5-$

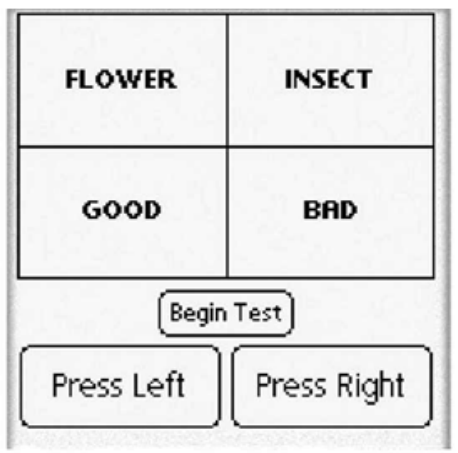

$-3-$

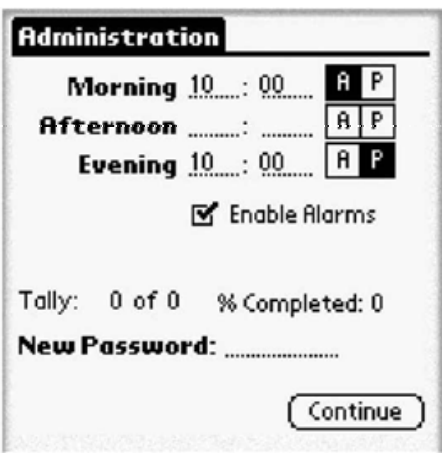

$-6-$

Figure 1. Palm IAT screens. Screens 1-4 are typical displays a subject sees during a testing session. Screens 5 and 6 are available only to the experimenter.

Palm IAT on a Handspring Visor PDA, and loaned them the PDA to keep for the duration of the study.

\section{Study 1: Flowers and Insects}

Study 1 replicated Greenwald et al.'s (1998) IAT demonstration of a preference for flowers over insects and examined the reliability of individual differences in this preference. We measured subjects' RTs in linking the concepts of flower and insect to the concepts of good and bad. The three stimulus words used to define each of these four concepts are listed in Table 1. All the subjects received the compatible set of word pairings ( flower-good and insectbad) before the incompatible set (flower-bad and insectgood).

Eight women and 4 men participated. They were given PDA's and were instructed to take the Palm IAT once each evening for 8 days; they actually took it a mean of 5.9 times each.
We downloaded the output files and analyzed them, using SAS. To reduce error, we dropped RT scores shorter than $400 \mathrm{msec}$ or longer than $1,400 \mathrm{msec}$. This is narrower than the 300 to 3,000 msec trimming window used by Greenwald et al. (1998), but we tried several windows and found that 400-1,400 msec produced the highest reliability (see below). Using the remaining data, we computed an IAT effect score, defined as the mean difference in RT to compatible (flower-good or insect-bad) versus incompatible (flower-bad or insect-good) word pairings, for each occasion on which a subject took the IAT. Overall mean RTs for compatible and incompatible conditions across all measurements were 751 and $852 \mathrm{msec}$, respectively, producing an overall mean IAT effect score of $101 \mathrm{msec}(S D=83 \mathrm{msec})$ and a statistical effect size of $d=1.22$. This is large in statistical terms, although smaller than the $223 \mathrm{msec}$ and $d=2.30$ reported by Greenwald et al. for a compatible-first IAT condition on a

Table 1

Stimulus Input File

FLOWER,INSECT,GOOD,BAD,daffodil,daisy,tulip,gnat,mosquito,roach,great,terrific,wonderful,awful, cancer,horrible

FLOWER,INSECT,BAD,GOOD,daffodil,daisy,tulip,gnat,mosquito,roach,awful,cancer,horrible, great, terrific,wonderful

Note-The file contains two lines of comma-delimited words for each IAT. Each line contains the four concept words that will appear, respectively, in the upper left, upper right, lower left, and lower right quadrants at the top of the PDA of the screen, followed by three exemplar words associated with each of them. 
Table 2

Response Output File

\begin{tabular}{|c|}
\hline $\begin{array}{l}840 \\
11 b\end{array}$ \\
\hline $\begin{array}{l}\text { Note-The file contains two lines of tab-delimited data for each IAT. Each line contains the subject's identi- } \\
\text { fication, the four concept words as they appeared in the input file, the date and time the test was taken, a num- } \\
\text { ber indicating the quasi-random order in which stimulus words were presented, } 24 \text { reaction times (RTs, in mil- } \\
\text { liseconds), and } 24 \text { scores }(0-1) \text { indicating whether a response was correct or incorrect. As more IAT tests are } \\
\text { run, more lines of data are added to the output file. In the example below, RTs are noticeably higher in the in- } \\
\text { compatible (flower-bad and insect-good) condition. }\end{array}$ \\
\hline
\end{tabular}

desktop computer. A between-subjects analysis of variance revealed significant differences among subjects in the IAT effect $[F(11,70)=3.03, p<.01]$. Although betweensubjects differences can be caused by differences in attention, comprehension, testing conditions, or experimental treatments, the most plausible interpretation here seems to be that some subjects preferred flowers over insects to a greater degree than did others.

We used the intraclass correlation coefficient to index the reliability of IAT measures of subjects' implicit preferences. The intraclass $r$ will be high to the extent that subjects differ among themselves in their preferences and react similarly on all measurement occasions. The intraclass $r$ was .25.

\section{Study 2: Agency and Communion}

In Study 2, we examined the reliability of Palm IAT as a measure of agency and communion. Agency refers to being independent and getting things done, and communion refers to forming relationships and helping other people (Bakan, 1966). We measured subjects' RTs in linking myself and other to the concepts of agency and com- munion, operationalizing agency and communion with the terms active versus passive and warm versus cold. The sets of 3 stimulus words we used to exemplify the concepts of myself, others, active, quiet, warm, and cold were, respectively, me-my-mine, they-them-their, activeassertive-energetic, quiet-hesitant-passive, warmfriendly-cooperative, and cold-hostile-aloof.

Eight women and 1 man participated. They were given PDAs and were instructed to take the Palm IAT when the PDA alarm went off at 10:00 a.m. and 10:00 p.m. each day for a period of 6 days; they actually took it a mean of 9.5 times each.

We trimmed the RT scores as above and created agency and communion IAT effect scores for each subject for each day's morning and evening measurements. For the agency IAT effect, we subtracted mean RT to the myselfquiet and other-active word pairings from mean RT to the myself-active and other-quiet word pairings. For the communion IAT effect, we subtracted mean RT to the myself-cold and other-warm word pairings from mean RT to the myself-warm and other-cold word pairings. Subject $\times$ time of day analyses of variance of the IAT ef-

Table 3

Reaction Times (in Milliseconds) to 12 Agency or Communion Words When Paired With the Concepts of Self or Other

\begin{tabular}{lccc}
\hline Attribute Word & Paired With Self & Paired With Other & Self - Other Difference \\
\hline High agency & & & \\
$\quad$ Active & 807 & 901 & -94 \\
$\quad$ Assertive & 835 & 934 & -99 \\
$\quad$ Energetic & 847 & 902 & -55 \\
High communion & & & \\
$\quad$ Warm & 788 & 887 & -99 \\
Friendly & 805 & 930 & -67 \\
Cooperative & 868 & 935 & \\
Low agency & & & 94 \\
$\quad$ Passive & 917 & 823 & -11 \\
Hesitant & 958 & 889 & 81 \\
Quiet & 904 & 915 & 73 \\
Low communion & & & 106 \\
$\quad$ Cold & 850 & 769 & \\
Hostile & 946 & 873 & \\
Aloof & 929 & 823 & \\
\hline
\end{tabular}

Note-Overall, subjects responded a mean of $90 \mathrm{msec}$ faster in pairing high agency and communion words with self than with other and $69 \mathrm{msec}$ faster when pairing low agency and communion words with other than with self. Overall $S D$ of the reaction time scores was $171 \mathrm{msec}$. 
fect scores revealed significant differences among subjects on both agency $[F(8,76)=5.92, p<.01]$ and communion $[F(8,76)=3.94, p<.01]$, indicating that the IAT can detect individual differences on these dimensions. There was also a subject $\times$ time of day communion interaction $[F(8,76)=2.26, p<.05]$, produced by a pattern of some subjects' implicitly associating themselves more strongly with communion in the morning than in the evening. Mean RTs in response to agency and communion words when paired with self and other words are shown in Table 3.

As in Study 1, we computed intraclass $r$ s to index the reliability of the IAT measures. The intraclass $r$ was .34 for agency and .24 for communion.

\section{Study 3: Boldness and Friendliness}

Study 3 examined reliability of Palm IAT data collected in an experiment on the effects of testosterone on feelings of boldness and friendliness. We measured subjects' RTs in linking myself and other to the concepts of boldness and friendliness. We used the same stimulus words as those in Study 2 for myself and other, and we operationalized boldness and friendliness by the terms bold versus shy and friendly versus hostile. The stimulus words used to exemplify bold, shy, friendly, and hostile were, respectively, active-energetic-bold, quiet-shy-cautious, warm-kindfriendly, and aggressive-combative-hostile.

Twelve women and 11 men participated. They were given PDAs and were instructed to take the Palm IAT in the morning and afternoon of 4 days; they actually took the test a mean of 6.7 times each.

We trimmed RT scores as above and created boldness and friendliness IAT effect scores for each subject for each measurement occasion. For the boldness IAT effect, we subtracted mean RT to the myself-shy and other-bold word pairings from mean RT to the myself-bold and other-shy word pairings. For the friendliness IAT effect, we subtracted mean RT to the myself-hostile and otherfriendly word pairings from mean RT to the myselffriendly and other-hostile word pairings. Between-subjects analyses of variance of the IAT effect scores revealed significant differences among subjects on both boldness $[F(22,130)=2.87, p<.001]$ and friendliness $[F(22,130)=$ $6.14, p<.001]$, indicating that the IAT can detect individual differences on these dimensions.

As above, we computed intraclass $r$ s to index the reliability of the IAT measures. The intraclass $r$ was .22 for boldness and .43 for friendliness.

\section{DISCUSSION}

Using a PDA device, we replicated a desktop computer IAT finding regarding a widely held attitude (more favorable evaluations of flowers than of insects) and found individual differences in associating the self with characteristics of agency, communion, boldness, and friendliness. The Palm IAT facilitates efficient data collection from large numbers of subjects, can be used in the absence of an experimenter, and can be taken outside the laboratory to study hard-to-reach populations in unusual settings. Although the paper-and-pencil version of the IAT (Lemm \& Banaji, 2000) has some of these advantages, it requires an experimenter and a quiet testing area and measures only a single aggregate RT response, rather than separate responses to individual items. As of yet, there have been no studies directly comparing responses on the Palm IAT with the desktop computer IAT or the paper-and-pencil IAT.

Reliability of measurements from the Palm IAT on repeated occasions was variable, ranging from .22 for the self-bold IAT to .43 for the self-friendly IAT, with a mean of .29. This is near the low end of the range of reliabilities reported for the desktop computer IAT, where Cunningham, Preacher, and Banaji (2001) found test-retest reliabilities for racial attitudes ranging from .17 to .50 , with a mean of .27, and Greenwald and Nosek (2001) reported reliabilities across several studies averaging above 60 . The low-to-modest reliability coefficients for the Palm IAT need not be a serious drawback, because reliability increases as the number of measurements accumulates, and it is relatively easy to accumulate measurements with the Palm IAT. The Spearman-Brown formula predicts reliability of the mean of several measurements from the reliability of a single measurement. Applying this formula, the reliability coefficient of .22 for the self-bold Palm IAT would increase to .59 for five repetitions of the measure, and a reliability of .43 for the self-friendly Palm IAT would increase to .79 for five repetitions of the measure. With the PDA device, it is relatively easy to make repeated measurements, combine them into an overall mean value, and thereby gain reliability. The issue of reliability should not be overemphasized, because this may presume that attitudes are stable and should be reflected similarly in different measurements over time, when in fact they are quite malleable. IAT scores have been shown to change, depending on experimenter characteristics (Lowery, Hardin, \& Sinclair, 2001), target context (Wittenbrink, Judd, \& Park, 2001), and priming (Dasgupta \& Greenwald, 2001). Some of what appears to be unreliability in the IAT scores may be an effect of this malleability.

Although test-retest reliability of the Palm IAT may be adequate, issues of construct validity raise serious concerns. The Palm IAT is limited to the use of three stimulus words to exemplify each concept. With so few words, it is difficult to capture the full meaning of a construct, and investigators may mislead themselves about what they are measuring. In the present studies, for example, the stimulus words for agency and communion were similar to the stimulus words for boldness and shyness. The same words might also define potency and warmth (Rudman, Greenwald, \& McGhee, 2001). Different investigators may use the same words for different concepts. The problem lies, in part, in the inherent overlapping definitions of constructs. The IAT is a somewhat blunt instrument for distinguishing among closely related concepts, and researchers who wish to examine the subtleties of a concept might be well advised to use some approach other than the IAT. 
A major advantage of the Palm IAT is that it lets researchers measure implicit attitudes in a variety of settings outside the laboratory. This makes it easier than before to measure implicit attitudes of people who are not represented in psychology subject pools or not available for laboratory testing, such as firefighters, medical workers, lawyers, engineers, transsexuals, shoppers, movie-goers, and criminals. Implicit attitudes about death could be measured by taking the Palm IAT to a funeral home, a funeral service college, or a morticians conference. The Palm IAT could be used to examine changes in attitudes that follow real-world events. A fan's change in self-concept or change in allegiance to a sports team could be measured at a ball park following an important win or loss. Other settings where one might use the Palm IAT include political rallies, protests, demonstrations, festivals, parades, graduation ceremonies, banquets, and the aftermath of fires, earthquakes, hurricanes, or tornados. The Palm IAT provides privacy of use, relatively low cost, and portability. Development of similar programs on the Palm platform is continuing, and we are currently preparing a Palm version of Nosek and Banaji's (2001) go/no-go association task.

\section{REFERENCES}

BAKAN, D. (1966). The duality of human existence. Chicago: Rand McNally.

Citrin, L. B., \& Greenwald, A. G. (1998, April). Measuring implicit cognition: Entomologists' and psychologists'attitudes toward insects. Poster presented at the annual meeting of the Midwestern Psychological Association, Chicago.

Cunningham, W. A., Preacher, K. J., \& Banaji, M. R. (2001). Implicit attitude measurement: Consistency, stability, and convergent validity. Psychological Science, 12, 163-170.
Dasgupta, N., \& Greenwald, A. G. (2001). On the malleability of automatic attitudes: Combating automatic prejudice with images of liked and disliked individuals. Journal of Personality \& Social Psychology, 81, 800-814.

Greenwald, A. G., McGhee, D. E., \& Schwartz, J.L.K. (1998). Measuring individual differences in implicit cognition: The implicit association test. Journal of Personality and Social Psychology, 74, 14641480 .

Greenwald, A. G., \& Nosek, B. A. (2001). Health of the Implicit Association Test at age 3. Zeitschrift für Experimentelle Psychologie, $\mathbf{4 8}$ 85-93.

Lemm, K., \& BANAJI, M. R. (2000, February). Motivation to respond without prejudice moderates the relationship between implicit and explicit prejudice. Poster presented at the annual meeting for the Society of Personality and Social Psychology, Nashville, TN.

Lowery, B. S., Hardin, C. D., \& Sinclair, S. (2001). Social influence effects on automatic racial prejudice. Journal of Personality \& Social Psychology, 81, 842-855.

NoseK, B., \& BANAJI, M. R. (2001). The go/no-go association task. Social Cognition, 19, 225-266.

Rudman, L. A., Greenwald, A. G., \& McGhee, D. E. (2001). Implicit self-concept and evaluative implicit gender stereotypes: Self and ingroup share desirable traits. Personality \& Social PsychologyBulletin, 27, 1164-1178.

Schiffman, S. (2000). Real-time self-report of momentary states in the natural environment: Computerized ecological momentary assessment. In A. A. Stone, J. S. Turkkan, C. A. Bachrach, J. B. Jobe, H. S. Kurtzman, \& V. S. Cain (Eds.), The science of self-report: Implications for Research and Practice (pp. 277-296). Mahwah, NJ: Erlbaum.

Whalen, C. K., Jamner, L. D., Henker, B., \& Delfino, R. J. (2001). Smoking and moods in adolescents with depressive and aggressive dispositions: Evidence from surveys and electronic diaries. Health Psychology, 20, 99-111.

Wittenbrink, B., Judd, C. M., \& PARK, B. (2001). Spontaneous prejudice in context: Variability in automatically activated attitudes. Journal of Personality \& Social Psychology, 81, 815-827.

(Manuscript received July 6, 2001; revision accepted for publication July 8, 2002.) 